\title{
Automatic elongated object segmentation in CT image using Stroke Width Transform and Levelset
}

\author{
Pi zhi-peng ${ }^{a}$, Wang zhi-ming ${ }^{b}$ \\ School of Computer \& Communication Engineering,University of Science \& Technology, Beijing \\ 100083, China \\ apzp147258@126.com bwangzhiming@ies.ustb.edu.cn,
}

Keywords: Stoke Width Transform; Elongated object; Levelset; Image segmentation

\begin{abstract}
In this paper, we propose a full-automatic algorithm for extracting elongated object from CT image. Given an input image, we use the SWT(Stroke Width Transform) and MM(Mathematical Morphology) to locate the coarse location of target. Thus leading to over-segmentation. In order to refine the segmentation result, Levelset method is applied to make the contour fit the object. Experimental results show that the proposed algorithm gives precise contour in elongated object segmentation.
\end{abstract}

\section{Introduction}

Detecting object in CT image is a vital step for a number of security inspection applications, such as explosive identification, weapon recognition. It is often difficult to extract object because of low contrast property and the effect of interferent. Image segmentation is a ill-posed problem and often needs to deal with fine-structure objects that hold the specific information. it is necessary to take advantage of target's shape prior knowledge for extracting the fine-structure. In security inspection, a lot explosives are thin elongated objects, in medical field, vessels are often long and thin. To extract these elongated objects in complex environment, it is essential to study approaches regarding how to effectively extract the fine-structure of the image[1].

A lot of methods have been proposed for different situations, and most segmentation algorithm can be divided into two main sub-steps: (1) detection and localization, (2) refinement and enhancement. In first step, MM is often used to find the target position. The result in Candeias' experiments shows that MM is superior to other edge-detection methods in extracting inner edge[2]. Peng eliminated a large chunk of background area by applying top-hat transform[3]. In order to relieve the effect of interferent, Andres Serna used a morphological h-maxima filter to get rid of maxima caused by texture information and noise on the upper part of the objects[4]. Wang calculated the location of nearest contour pixel for each pixel, which is used to generate a more accurate skeleton[5]. Guillaume separated the image and text part from document image with connected filter[6].

After the coarse localization, a more precise method can be applied to previous result for refining the segmentation. Osher proposed Levelset method for handling the topologic problem[7] in Active Contour Model. Although this algorithm is of great characteristics, some drawbacks, like time-consuming problem and troublesome initialization, limit its usage. To solve these problems, Chan proposed a new energy based on statistic property, thus making Levelset more stable for different initialization[8]. Liu added local energy to C-V Model for speeding up the segmentation[9]. re-initialization is a time-consuming step during contour evolving, Li removed this step by adding another energy cost, which reduced the time of iteration greatly[10].

In this paper, we present a new algorithm by combining Stroke Width Transform(SWT) and Levelset method. The main purpose is to extract elongated object in CT image. We first use SWT and MM to find the position of elongated object, and then Levelset method is applied to refine the segmentation result. 


\section{Previous Work}

\section{Strokes Width Transform Model.}

SWT is proposed by Epshtein for locating text position on image. The basic idea of this transform is that every pixel on image owns a stroke with a specific stroke width, text pixels usually have nearly constant stroke width. SWT merges pixels of similar stroke width into larger components. We can find the most likely text are by bottom up integration[11, 12].

Stroke width of each pixel on image is based on the gradient vector of pixel, we search from current pixel along the direction of inverse gradient, and stop immediately when encounter a edge pixel. The distance from the last pixel to start pixel is calculated and stored for pixels we visited before(Fig. 1).

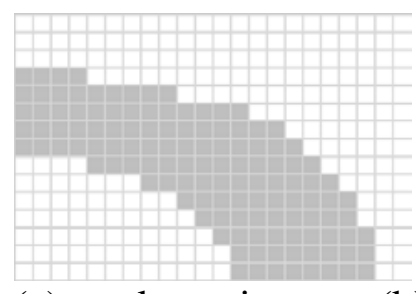

(a)a stoke on image (b)search from p to q, width w is calculated

Fig. 1 principle of SWT

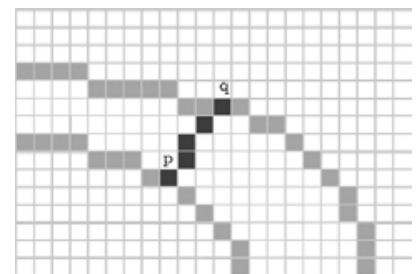

(c)mark all pixels with $\mathrm{w}$

For pixels of foreground, the distance of each pixel is updated according to the path passing through it. We can use the minimum path length to represent its stoke width, but the distance of pixels near corner can be updated to a incorrect value, so Epshtein proposed a solution to solve this problem: replace the minimum value with the median stoke width along the searching direction, this method can correct the false distance to some extent.

\section{Elongated object segmentation}

The first step for elongated object segmentation is locating the potential area. We use the modified SWT to find the rough object on image, the result is refined by MM, and Levelset method will be applied to the coarse result for obtaining a final segmentation result.

\section{Modified Stroke Width Transform.}

A distance map created by SWT[12] will be used for pixels merging and we can see that incorrect merging result is generated (Fig. 2(c)) because of some pixels being never updated to a right distance. To overcome this problem, neighbor pixels of searching path are tested. This paper uses the following formula to update a right distance into distance map:

$$
\mid \operatorname{dot}(\operatorname{norm}(P-q) \text {, norm }(\text { dir })) \mid<\text { threshold }
$$

Where dot means dot product of two vector, norm means normalizing a vector, $P$ is the position of current pixel, while $q$ is the position of test pixel, dir represents inverse gradient of current pixel. The value of threshold is 0.4 in our experiments.

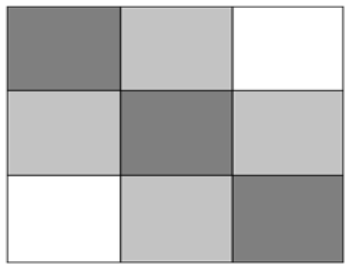

(a)neighbor pixels

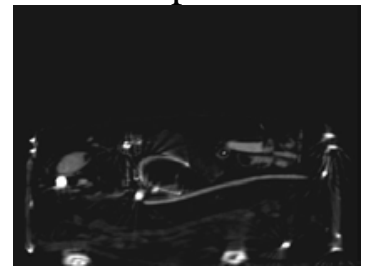

(b)original image
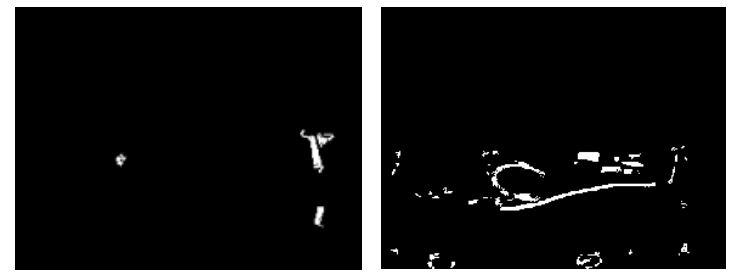

(d)result of our method

Fig. 2 generate distance map

In order to gain a good segmentation result, we create a histogram for distance map, and remove the pixels with too little or too large distance value. The result is showed in Fig. 2(d). According to the SWT and the shape prior of CT image, we can suppose that target contain the maximum pixels with the same stroke width, thus leading us to find the most likely searching area, and the stroke width containing maximum pixels should be the stoke which we are looking for. 
Neighbor pixels are considered which result in an over segmentation, thus may lead to incorrect stoke branches, we propose a simple pruning method to solve this problem. The skeleton is extracted by mathematical morphology operation(see Fig. 3).

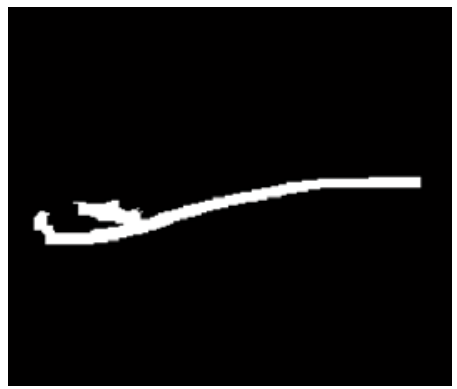

(a)extraction result

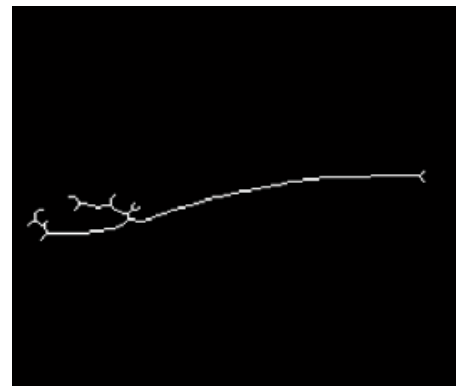

(b)skeleton of (a)

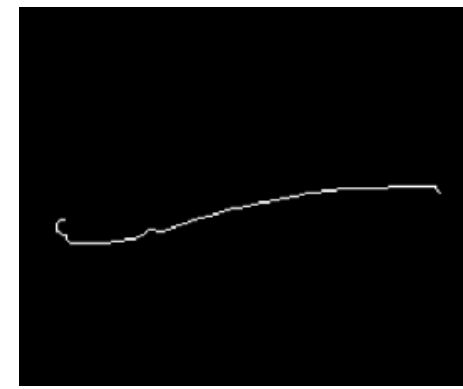

(c)result of prune operation

Fig. 3 remove incorrect branches

There are many incorrect branches in Fig. 3(b), and these false branches are removed in Fig. 3(c), the pruning method is described in detail below:

Algorithm 1 the proposed algorithm to prune the skeleton

Step1. Find all endpoints $v[n], n$ is the number of endpoints, all skeleton pixel has a marked value 1 , while 0 for background.

Step2. Search from endpoints $\mathrm{v}[\mathrm{n}]$, for endpoint $e$ and its skeleton neighbor pixel $b$, update marked value: $b=1+e$, move $e$ to $b$.

Step3. If the endpoint in $\mathrm{v}[\mathrm{n}]$ meets the main branch of skeleton(there are more than 1 neighbor skeleton pixels), remove it from $v[n], n=n-1$.

Step4. If $\mathrm{n}$ equal to $\mathrm{k}$ ( the number of target object's endpoints, $\mathrm{k}$ is 2 for our target), jump to Step5, or back to Step2.

Step5. Mark the foreground pixels that we haven't touched as bone pixel. For each point $e$ in $\mathrm{v}[\mathrm{k}]$, find its adjacent pixel with the maximum marked value, move $e$ to this pixel, and mark this pixel with value 1 . Record this pixel.

Step6. Stop finding if there is no pixel whose marked value larger than 1.

Step7. Mark recorded pixels as bone pixels. End.

We now get a main skeleton of object, then a dilation operator is applied to this skeleton image(according to the stroke width gained before).

\section{Levelset Processing.}

The dilation result can fit the object's outline coarsely, and to refine the segmentation, we applied Levelset method[9, 13, 14] to coarse segmentation result, and the basic Levelset energy function is:

$$
E\left(c_{1}, c_{2}, C\right)=\lambda_{1} \int_{\operatorname{in}(C)}\left(I(x)-C_{1}\right)^{2} d x+\lambda_{2} \int_{\text {out }(C)}\left(I(x)-C_{2}\right)^{2} d x+\mu \cdot \operatorname{Length}(C)+v \cdot \operatorname{Area}(\operatorname{in}(C))
$$

Where $\mathrm{C}$ is current contour, $\mathrm{c}_{1}$ and $\mathrm{c}_{2}$ means average intensity of inner and outer side of contour, parameters $\lambda_{1}, \lambda_{2}, \mu$ and $v$ are fixed coefficient. Fig. 4 shows the Levelset result.
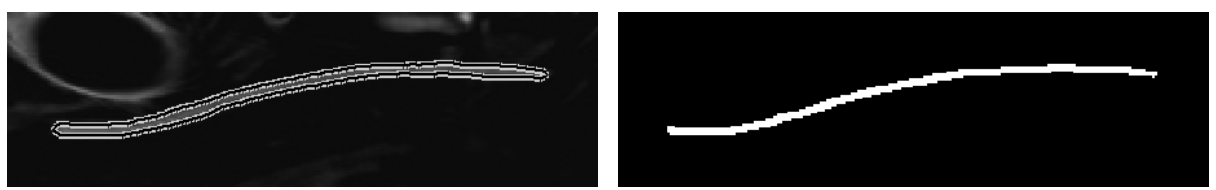

Figure 4 Levelset initialization(left) and segmentation result(right)

Levelset method is easy to evolve to incorrect contour position in CT image, so the dilation result is introduced for restricting the boundary of Levelset area, we calculate their intersection set to remove incorrect area. 


\section{Experiment result}

\section{Comparison.}

Fig. 5 shows a comparison among Levelset, GrowCut[15], Maximum Entropy Method[16], and our method. To make Levelset and GrowCut work properly, we initialize the contour manually, while our method can segment the image automatically.

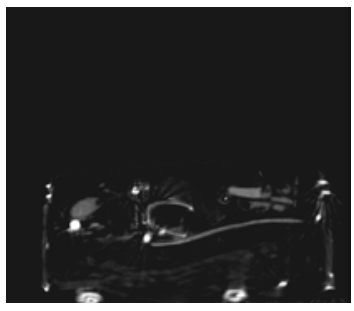

(a)original image

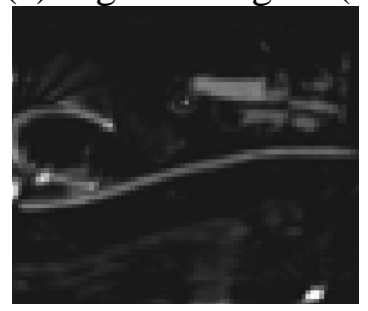

(d)target object

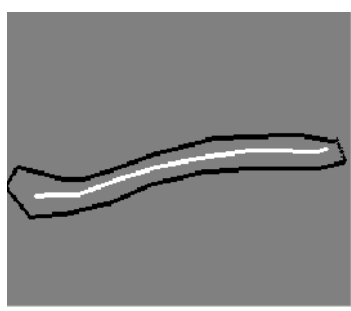

b)initialization for (e)

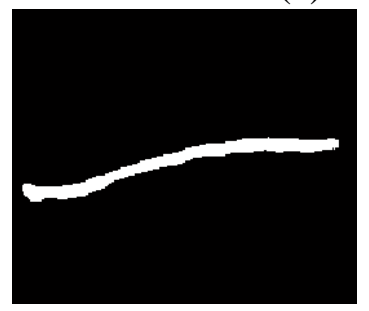

(e)GrowCut

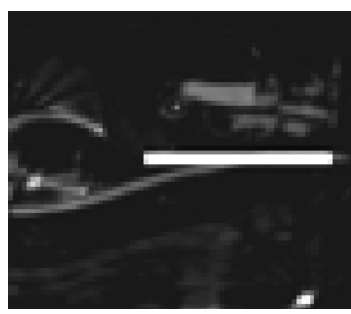

(c)initialization for (f)

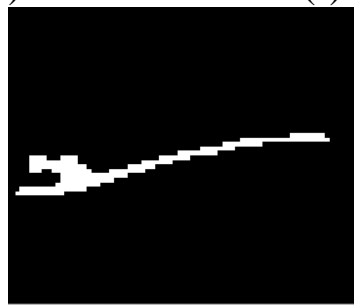

(f)Levelset
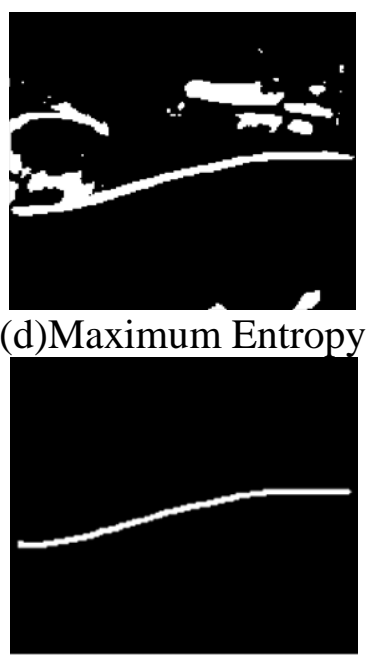

(g)our method

Fig. 5 experiment result

We can see that the proposed method removed incorrect branches while Maximum Entropy and Levelset failed, the final contour of our method is more smooth than others.

\section{Analysis.}

We use F-Measure to evaluate the segmentation result of these method, F-Measure assesses result with the ground truth image created manually, it is defined as below[1]:

$F=2 R P / P+R$

Where $P$ is precision value and $R$ is recall value. Table 1 shows the scores of methods we mentioned before. and our method give the most precise result and it is less time-consuming.

Table 1 Performance of different methods

\begin{tabular}{|l|l|l|l|l|}
\hline Algorithm & Precision & Recall & F-Measure & Time(s) \\
\hline GrowCut & 0.5556 & 0.9824 & 0.7097 & 23.12 \\
\hline Levelset & 0.5739 & 0.8729 & 0.6925 & 11.52 \\
\hline Maximum Entropy & 0.1332 & 0.8368 & 0.2298 & 1.45 \\
\hline Our method & 0.9148 & 0.6295 & 0.7458 & 5.14 \\
\hline
\end{tabular}

\section{Conclusion}

In this paper, we propose a new algorithm which combines SWT model and MM for initializing the contour automatically. This method restricts the searching area of Levelset, thus lowering the time of iteration, and incorrect branches are removed by pruning step, then Levelset method is applied for refining the segmentation. Experimental results show that the proposed algorithm is able to segment elongated object effectively in CT image.

\section{References}

[1] Zhu Q, Ye R, Shao L, et al. A novel thin elongated objects segmentation based on fuzzy connectedness and GMM learning[C]. ICIP, Melbourne. 2013: 4273-4276.

[2] Candeias A L B, Nascimento P H O, Junior J R T, et al. Edges Extraction with Mathematical Morphology Tools and Canny Filter: A Comparison[J]. Open Journal of Computer Science and 
Engineering Survey, 2015, 3(1): 62-70.

[3] Peng Zhi-hao, Yang Feng-bao, Wang Zhi-She, et al. Infrared Target Extraction Based on Mathematical Morphology and Automatic Region Growing[J]. Infrared Technology, 2014, 36(1): 47-52.

[4] Serna A, Marcotegui B. Detection, segmentation and classification of 3D urban objects using mathematical morphology and supervised learning[J]. Journal of Photogrammetry and Remote Sensing, 2014, 93: 243-255.

[5] Wang Jin-Ling. Research on Skeleton Pruning Algorithom with Continuty[D]. Shandong Normal University, 2010.

[6] Lazzara G, Géraud T, Levillain R. Planting, growing and pruning trees: Connected filters applied to document image analysis[C]. Document Analysis Systems, Tours, 2014: 36-40.

[7] Osher S, Sethian J A. Fronts propagating with curvature-dependent speed: algorithms based on Hamilton-Jacobi formulations[J]. Journal of computational physics, 1988, 79(1): 12-49.

[8] Chan T F, Vese L A. Active contours without edges[J]. Image processing, IEEE transactions on, 2001, 10(2): 266-277.

[9] Liu S, Peng Y. A local region-based Chan - Vese model for image segmentation[J]. Pattern Recognition, 2012, 45(7): 2769-2779.

[10]Li C, Xu C, Gui C, et al. Distance regularized level set evolution and its application to image segmentation[J]. Image Processing, IEEE Transactions on, 2010, 19(12): 3243-3254.

[11] Jameson J, Abdullah S N H S. Extraction of arbitrary text in natural scene image based on stroke width transform[C]. Intelligent Systems Design and Applications, International Conference on. IEEE, 2014:124-128.

[12]Epshtein B, Ofek E, Wexler Y. Detecting text in natural scenes with stroke width transform[C]. Computer Vision and Pattern Recognition, San Francisco, 2010: 2963-2970.

[13]Kass M, Witkin A, Terzopoulos D. Snakes: Active contour models[J]. International journal of computer vision, 1988, 1(4): 321-331.

[14]Li C, Xu C, Gui C, et al. Level set evolution without re-initialization: a new variational formulation[C]. Computer Vision and Pattern Recognition, San Diego, 2005: 430-436.

[15]Chen J M, Lu H Y. Color Image Segmentation Based on Secondary Watershed and GrowCut Algorithm[J]. Advanced Materials Research, 2014, 989:4032-4037.

[16]Peng Wu. Image segmentation method based on firefly algorithm and maximum entropy method[J]. Computer Engineering \& Applications, 2014, 50(12):115-119. 